\title{
FATORES DE RISCO CARDIOVASCULARES EM TRABALHADORES DE ENFERMAGEM EM UM CENTRO DE REFERÊNCIA NO SUL DO BRASIL ${ }^{1}$
}

\section{FACTORS OF HEART ATTACK RISKS IN WORKERS OF NURSING IN A CENTER OF REFERENCE IN SOUTHERN BRAZIL}

\author{
FACTORES DE RIESGO CARDIOVASCULAR EN \\ TRABAJADORES DE ENFERMERÍA EN UN CENTRO DE \\ REFERENCIA EN EL SUR DE BRASIL
}

\author{
Claudiane Bottoli ${ }^{*}$ \\ Ma Antonieta Moraes** \\ Silvia GoldmeIER ${ }^{* * *}$
}

\begin{abstract}
RESUMO
Introdução: Atualmente as doenças cardiovasculares, em especial, a doença arterial coronariana (DAC) representa 35\% das causas de morte das doenças vasculares. Objetivos: Identificar os fatores de risco (FR) modificáveis para doença arterial coronariana em trabalhadores de enfermagem que atuam em Pronto Socorro, Centro Obstétrico, Centro Cirúrgico e Unidades de Terapia Intensiva (Pediátrica, Adulta, Neonatal); correlacionar os resultados entre as categorias de enfermagem, turnos e setores de trabalho. Métodos: Estudo transversal contemporâneo. Foi aplicado um questionário com finalidade de rastrear fatores de risco, pressão arterial (PA), dados antropométricos, glicemia de jejum, colesterol total- Hdl e Ldl, triglicerídeos e aplicação do inventário de sintomas de estresse de adultos de LIPP ( ISSL). Resultados: Foram incluídos 80 participantes do estudo, $86 \%$ eram mulheres com idade média de $37,7 \pm 8,2$ anos, $26 \%$ eram enfermeiros, $56 \%$ eram técnicos de enfermagem e $17 \%$ eram auxiliares de enfermagem. O setor da UTI prevaleceu em número de trabalhadores (45\%) e o diurno foi o turno predominante $(41,2 \%)$. Os FR mais relevantes foram história familiar $(86,3 \%) \mathrm{em}$ $1^{\circ}$ grau de parentesco, o sedentarismo (55\%), colesterol total alto $(6,3 \%)$ e limítrofe $(26,3 \%)$,estresse $(53,8 \%)$ e o sobrepeso (56,3\%). Conclusão: Nosso estudo mostrou que esta população possui risco aumentado para DCV. O acompanhamento desta amostra poderá nos trazer resultados surpreendentes por tratar-se de uma população jovem com prevalência de resultados limítrofes. Programas deverão ser adotados para auxiliar a promoção à saúde e a mudança do estilo de vida iniciando precocemente, na infância.
\end{abstract}

Palavras chave: Doença arterial coronariana, fatores de risco, trabalhadores de enfermagem.

\footnotetext{
${ }^{1}$ Monografia apresentada ao Programa de Pós-Graduação Latu senso em Enfermagem do IC/FUC.

*Enfermeira, Especialista em Cardiologia pelo Programa de Pós-graduação latu sensu Enfermagem em Cardiologia do IC/ FUC. editoração-pc@cardiologia.org.br - Enfermeira Assistencial do Hospital.

${ }^{* *}$ Universitário da Universidade Federal de Santa Maria - HUFSM. Enfermeira, Mestre em Ciências da Saúde pelo IC/FUC. antonieta_moraes@uol.com.br - Professora do Programa de Pós-graduação latu sensu em Cardiologia do IC/FUC.

*** Enfermeira, Mestre em Ciências da Saúde pelo IC/FUC. silvia.gold@cardiologia.org.br - Professora do Programa de Pósgraduação latu sensu em Cardiologia do IC/FUC. Unidade de Pesquisa do IC/FUC.

Av. Princesa Isabel, 370 Santana, Porto Alegre, RS, Brasil 90620-000 Fone/Fax.: +55+51+3219-2802 ext.: 12, 23.editoracaopc@cardiologia.org.br
} 


\begin{abstract}
Introduction: Nowadays the cardiovascular diseases (CVD), specially the Coronary Heart Disease (CHD), or Coronary Artery Disease (CAD), represent 35\% of causes of death of vascular diseases. Objectives: To identify the risk factors that can be modified for CHD/CAD in Nursing Workers who act at the Emergency Room, Obstetric Center, Center and Surgical Intensive Care Units (Pediatric, Adult, Neonatal); to correlate these results with the different categories of nursing as well as with their work shifts and sectors. Methods: Cross-study analysis. A questionnaire was applied aiming at tracking risk factors, blood pressure, anthropometric data, fasting glycemia, total cholesterol (HDL and LDL), triglycerides, as well as Lipp's Inventory of Stress Symptoms in Adults. Results: Out of 80 participants of the study, $86 \%$ were women aged about 37,7 $\pm 8,2$ years; $26 \%$ were Nurses, $56 \%$ were Nursing Technicians and $17 \%$ were Nursing Assistants. Most Nursing workers belong to the sector of the Intensive Care Unit ( $45 \%)$ and the daytime was the predominant shift (41,2\%). The most relevant risk factors were the family history (at 1st degree) $(86,3 \%)$, the sedentarism $(55 \%)$, total high cholesterol $(6,3 \%)$ and borderline cholesterol level $(26,3 \%)$, stress $(53,8 \%)$ and overweight $(56,3 \%)$. Conclusion: Our study has shown that population presents an increased CVD risk. Monitoring this sample can reveal surprising results in the future since it refers to a young population with predominance of bordering results. Programs should be adopted to facilitate health promotion towards a healthier life style since early in the childhood.
\end{abstract}

Key words: Coronary artery/heart disease, risk factors, nursing workers.

\title{
RESUMEN
}

Introducción: Actualmente las enfermedades cardiovasculares (ECV), en especial la enfermedad arterial coronaria (EAC), representan 35\% de las causas de muerte de las enfermedades vasculares. Objetivos: Identificar los factores de riesgo (FR) modificables para la enfermedad arterial coronaria en los trabajadores de enfermería que actúan en la Sala de Emergencia, Centro Obstétrico, Centro Quirúrgico y las Unidades de Cuidados Intensivos (pediátrico, adulto, neonatal); correlacionar los resultados entre las categorías del oficio de enfermería, los turnos y los sectores de trabajo. Métodos: Estudio transversal contemporáneo. Se aplicó un cuestionario para identificar los factores de riesgo, la presión arterial, los datos antropométricos, la glicemia en ayunas, el colesterol total (HDL y LDL), los triglicéridos; también se aplicó el inventario de síntomas del estrés de los adultos, de Lipp. Resultados: De los 80 participantes del estudio, $86 \%$ eran mujeres con la edad media de $37.7 \pm 8.2$ años; $26 \%$ eran enfermeros; $56 \%$ eran técnicos de enfermería y $17 \%$ eran auxiliares de enfermería. El sector de UTI prevaleció en número de trabajadores (45\%) y el diurno era el turno predominante (41.2\%). Los factores de riesgo más prevalentes eran la historia familiar $(86.3 \%)$ en primero grado de relación, el sedentarismo (55\%), el colesterol total alto (6.3\%) y el colesterol limítrofe (26.3\%), el estrés (53.8\%) y el exceso de peso (56.3\%). Conclusión: Nuestro estudio ha mostrado que la población presenta un FR aumentado de CVD. El monitoreo de esta muestra puede revelar resultados sorprendentes en el futuro ya que se refiere a una población joven con el predominio de resultados limítrofes. Deben ser adoptados programas para facilitar la promoción de salud hacia un estilo de vida más sano desde temprano en la infancia.

Palabras clave: Enfermedad arterial coronaria (EAC), factores de riesgo (FR), trabajadores de enfermería.

Fecha recepción: 19/10/07 Fecha aceptación: 06/08/09

\section{INTRODUÇÃO}

As doenças cardiovasculares (DCV) revelamse como a principal causa de mortalidade e morbidade (1). No Brasil estima-se que elas sejam responsáveis por aproximadamente $20 \%$ dos óbitos em sujeitos a partir dos vinte anos de idade (2). No RS a taxa de mortalidade atinge os $35,3 \%$ (3). Entre as DCV, destaca-se a doença arterial coronariana que ocasiona 35\% de mortalidade, sendo ocasio- 
nada por diversos Fatores de Risco (FR) que podem ser classificados como modificáveis e não modificáveis. Os modificáveis são dislipidemia, hipertensão arterial, tabagismo, diabetes mellitus (DM), obesidade, estresse $(1,2-4)$.

Entre os anos de 1975 e 1989 houve um aumento significativo da obesidade na população em geral. Um estudo realizado com 43 adolescentes com história familiar positiva para hipertensão (HAS), cujos pais eram hipertensos e normotensos, teve como objetivo avaliar hábitos alimentares, perfil lipídico, pressão arterial e dados antropométricos. Tal estudo identificou nos adolescentes filhos de hipertensos, que a pressão arterial sistólica (PAS) a pressão arterial diastólica (PAD)e o perfil lipídico eram mais elevados em comparação aos outros adolescentes (3).

\section{PROBLEMA}

Os cuidados com a saúde tem sido utilizado como medida para interpretar resultados de comorbidades e mortalidade. Diante disso busca-se a realização de um estudo com a finalidade de identificar os FR para DAC entre os trabalhadores da saúde-enfermagem que podem interferir na sua prática diária de vida.

\section{OBJETIVO}

Identificar os fatores de risco (FR) modificáveis para doença arterial coronariana em trabalhadores de enfermagem que atuam de Pronto Socorro, Centro Obstétrico, Centro Cirúrgico e Unidades de Terapia Intensiva (Pediátrica, Adulta, Neonatal).

Correlacionar os resultados dos fatores de risco entre as categorias de enfermagem, os turnos de trabalho e os respectivos setores que atuam.

\section{MATERIAL E MÉTODO}

Trata-se de um estudo transversal contemporâneo. A investigação foi realizada no Hospital Universitário da Universidade Federal de Santa Maria-HUSM. A população da pesquisa foi composta por trabalhadores de enfermagem (enfermeiros, auxiliares e técnicos) que atuam nos setores de Pronto Socorro, Centro Obstétrico, Centro Cirúrgico e Unidades de Terapia Intensiva (Pediátrica, Adulta, Neonatal). Dos 109 trabalhadores, 80 participaram da pesquisa. Foram excluídos os trabalhadores das unidades de internação e os que não concordaram em participar do estudo. O projeto foi encaminhado ao comitê de ética em pesquisa da instituição, e os profissionais incluídos assinaram o termo de consentimento livre e esclarecido (TCLE). O entrevistador apresentou o estudo aos trabalhadores, enfatizando o sigilo das informações, bem como a relevância do tema, e após foi aplicado um questionário, com a finalidade de investigar os fatores de risco para doença arterial coronariana.

As variáveis analisadas do estudo foram: idade, sexo, categoria profissional, setor de trabalho, nível de estresse, pressão arterial, obesidade, tabagismo, hipercolesterolemia e glicemia.

Os valores de pressão arterial foram aferidos conforme $\mathrm{V}$ Diretrizes Brasileiras de Hipertensão Arterial (Sociedade Brasileira Cardiologia, 2006). Utilizou-se um esfigmomanômetro do tipo aneróide e estetoscópio, previamente calibrado e validado pelo INMETRO. Foi considerado hipertenso, o trabalhador com uma pressão arterial sistólica (PAS) $\geq 140$ mmHg e pressão arterial diastólica $(\mathrm{PAD}) \geq 90 \mathrm{mmHg}$ (5).

As medidas antropométricas como peso, estatura e circunferência abdominal (CA) foram verificados para o cálculo do IMC e avaliação do risco aumentado (RA) para doença cardiovascular, com o auxílio de uma balança calibrada pela própria instituição. 
Para a verificação do peso, os trabalhadores permaneciam descalços, sem uso de casacos ou agasalhos. Os valores obtidos foram registrados em quilogramas. A estatura foi verificada com os profissionais eretos e com os calcanhares unidos, através da régua antropométrica da própria balança, e a circunferência abdominal medida com uma fita métrica acima da cicatriz umbilical. $\mathrm{O}$ risco aumentado para desenvolver DCV foi definido como $\mathrm{CA}>94 \mathrm{~cm}$ nos homens e CA $>88 \mathrm{~cm}$ nas mulheres (6). O IMC foi obtido através da divisão da massa corporal, em quilogramas, pelo quadrado da estatura, em metros. Foi considerado sobrepeso quando IMC entre 25 e $29,9 \mathrm{~kg} / \mathrm{m}^{2}$ e obesidade quando IMC $>30 \mathrm{~kg} / \mathrm{m}$ (2-6).

Os resultados dos exames laboratoriais referiam-se ao último periódico de saúde do trabalhador (novembro de 2005 a maio de 2006)que incluía exames de colesterol total, HDL, LDL, triglicerídeos e glicemia de jejum. Os valores de referencia foram: colesterol total $(\mathrm{CT})<200 \mathrm{mg} / \mathrm{dl}=$ normal, 200 a $239 \mathrm{mg} / \mathrm{dl}=$ limítrofe, $\geq 240 \mathrm{mg} / \mathrm{dl}=$ alto; triglicerídeos $<200 \mathrm{mg} / \mathrm{dl}=$ normal, $200 \mathrm{a}$ $400 \mathrm{mg} / \mathrm{dl}=$ limítrofe alto, $>400$ a $1000 \mathrm{mg} /$ $\mathrm{dl}=$ alto e $>1000 \mathrm{mg} / \mathrm{dl}=$ muito alto $(7)$.

$\mathrm{O}$ alcoolismo foi definido como a ingestão habitual e diária de bebida alcoólica distribuída ao longo do dia em várias doses (8).

Foi considerado como tabagista, aquele trabalhador que fumava qualquer quantia de cigarro diariamente ou aquele que havia deixado de fumar por tempo mínimo de um ano (8).

Foram considerados diabéticos os trabalhadores que apresentaram glicemia de jejum igual ou superior a $126 \mathrm{mg} / \mathrm{dl}$ (9) .

Para avaliarmos o nível de estresse, os trabalhadores responderam ao teste Inventário de sintomas de estresse para adultos de LIPP, de forma individual. Os testes foram analisados por um psicólogo devidamente habilitado, sendo classificados de acordo com a fase apresentada: alerta, resistência, quase exaustão e exaustão (10).
A atividade física foi considerada sedentária quando a prática era inferior a 2 vezes por semana, leve de 2 a 4 vezes por semana, moderada 5 a 7 vezes por semana, e de atividade física intensa quando a prática era diária (11).

\section{RESULTADOS}

Os resultados foram inseridos em uma planilha do Programa Excel for Windows. As análises foram realizadas utilizando o programa Statistical Package for Social Sciences (SPSS) 14.0. As variáveis categóricas foram expressas em percentual ou valor absoluto, as contínuas como média \pm desvio padrão ou mediana. Para as análises comparativas foi usado o teste Anova, e para as quantitativas e categóricas o Qui-quadrado. Foram considerados significativos um $\mathrm{p}<0,05$.

Foram incluídos no estudo 80 profissionais de enfermagem dos 109 admitidos através de concurso público no Hospital Universitário da Universidade Federal de Santa Maria. A média de idade da população estudada foi de 37 anos. Nossos resultados demonstraram que $56 \%$ da população incluída foram técnicos de enfermagem, 26\% enfermeiros e $17 \%$ auxiliares de enfermagem. Predominou trabalhadores da UTI (45\%), e do turno diurno $(41,2 \%)$. Dados demonstrados na Tabela 1.

\section{Fatores de risco}

Os fatores de risco mais prevalentes na nossa amostra foram a história familiar $(86,3 \%)$ em $1^{\circ}$ grau de parentesco como o mais preponderante. $\mathrm{O}$ sedentarismo esteve presente em 55\%. O colesterol alto e limítrofe apresentaram, respectivamente 6,3 e $26,3 \%$, e os triglicerídeos elevados $1,3 \%$ e limítrofe alto em $8,8 \%$. O estresse esteve presente em 53,8\% dos trabalhadores. Ao avaliarmos o IMC, nossos achados demonstraram que a obesidade e o sobrepeso esti- 
Tabela 1. Características da População $(\mathrm{n}=80)$.

\begin{tabular}{|c|c|c|c|}
\hline Características & $\mathrm{N}$ & $(\%)$ & Média \pm DP \\
\hline Idade (anos) & & & $37,7 \pm 8,2$ \\
\hline Sexo Feminino & 69 & $(86,3)$ & \\
\hline \multicolumn{4}{|l|}{ Categoria Profissional } \\
\hline Auxiliares & 14 & $(17,5)$ & \\
\hline Técnicos & 45 & $(56,2)$ & \\
\hline Enfermeiros & 21 & $(26,3)$ & \\
\hline \multicolumn{4}{|l|}{ Setor de Trabalho } \\
\hline Pronto Socorro & 18 & $(22,5)$ & \\
\hline Centro Obstétrico & 6 & $(7,5)$ & \\
\hline Centro Cirúrgico & 20 & $(25,0)$ & \\
\hline UTI & 36 & $(45,0)$ & \\
\hline \multicolumn{4}{|l|}{ Turno de Trabalho } \\
\hline Diurno & 33 & $(41,2)$ & \\
\hline Noturno & 25 & $(31,3)$ & \\
\hline Variado & 22 & $(27,5)$ & \\
\hline
\end{tabular}

Variáveis contínuas: média \pm desvio padrão e variáveis categóricas: porcentagens (\%).

Tabela 2. Prevalência de fatores de risco.

\begin{tabular}{lc}
\hline Fatores de risco & $\mathrm{n}(\%)$ \\
\hline HF de $1^{\circ}$ Grau & $83,6(69)$ \\
HAS & $9(11,3)$ \\
DM & $3(3,8)$ \\
Colesterol & \\
$\quad$ Alto & $21(26,3)$ \\
$\quad$ Limítrofe & $21(26,3)$ \\
Triglicerídeos $\quad$ & 1,3 \\
$\quad$ Alto & $7(8,8)$ \\
$\quad$ Limítrofe & $5(6,3)$ \\
Tabagismo & $41(5,3)$ \\
Mensumo eventual de álcool & 11 \\
Estresse & $43(53,8)$ \\
Sedentarismo & $44(55)$ \\
IMC & $60(75)$ \\
Obeso & $10(12,5)$ \\
Sobrepeso & $10(12,5)$ \\
\hline
\end{tabular}

HF: história familiar; HAS: hipertensão arterial sistêmica;. DM: diabete melito; Variáveis categóricas: porcentagens (\%); IMC: índice de massa corpórea.

veram em $56,3 \%$ da amostra, confirmando o risco aumentado para DCV em $75 \%$ através das medidas de CA. Dados demonstrados na Tabela 2.

Fatores de risco e categoria profissional Ao correlacionarmos os fatores de risco mo- dificáveis com as categorias profissionais, nossos resultados não demonstraram diferença estatisticamente significativa entre os profissionais de enfermagem. Percebe-se, porém, que o colesterol total teve um valor maior entre os auxiliares de enfermagem. Dados ilustrados na Figura 1. 
Figura 1: Fatores de risco e categoria profissional.

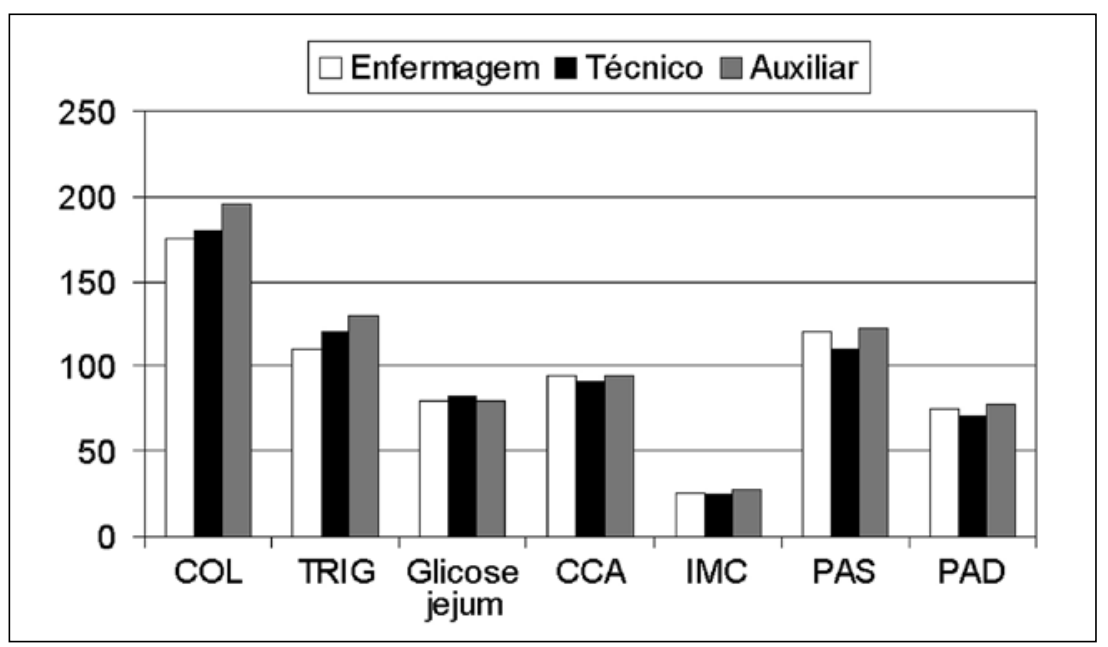

\section{Estresse}

O estresse foi analisado isoladamente e correlacionado ao IMC. Nossos achados demonstraram que 37(53\%) dos trabalhadores classificados em uma das fases de estresse também apresentaram obesidade $(60 \%)$ ou sobrepeso $(71,4 \%)$.

Ao ser comparado aos diferentes setores de trabalho, pode-se constatar que o estresse esteve presente em 11(61,1\%) dos trabalhadores do Pronto Socorro, 10(50,\%) no Centro Cirúrgico e 22(61,1\%) nos trabalhadores da Unidade de Terapia Intensiva. Nos trabalhadores alocados no Centro Obstétrico não houve registro de estresse.
Quando associamos o estresse entre as categorias profissionais utilizamos o teste inventário de sintomas de estresse para adultos, de LIPP, e foram classificados de acordo com a fase alerta, resistência, quase exaustão e exaustão. Nossos resultados demonstraram que os enfermeiros encontravam-se nas fases de quase-exaustão $(44,6 \%)$, exaustão $(42,3 \%)$, sintomas físicos (42,7\%) e sintomas psicológicos $(41,83 \%)$. Os técnicos de enfermagem prevaleceram na fase de resistência $(44,1 \%)$, sendo que os auxiliares de enfermagem mantiveram, em quase todas as fases, em níveis inferiores aos demais profissionais. Dados ilustrados na Figura 2.

Figura 2: Fases do estresse de acordo com a categoria profissional.

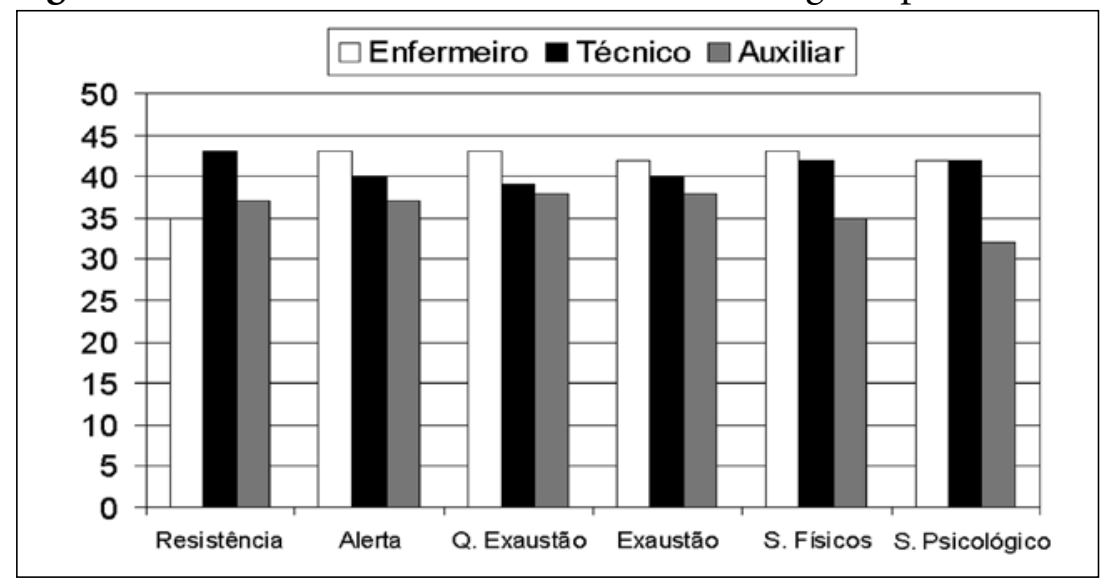




\section{DISCUSSÃO}

A amostra representa $40 \%$ dos trabalhadores lotados nos setores em estudo. A predominância do sexo feminino $(86,3 \%) \mathrm{em}$ nosso estudo confere que os espaços ocupados em hospitais, no atendimento direto ao paciente, são caracterizados como espaços femininos, cuja força de trabalho representa mais de $90 \%$ composta por mulheres (12). A média de idade dos trabalhadores manteve-se em índices inferiores a 40 anos motivado por recente concurso público cujo critério de inscrição não deveria ultrapassar 35 anos e cujas vagas eram destinadas ao local do estudo.

Entre os FR, percebeu-se que mais de $2 / 3$ dos sujeitos pesquisados possuíam antecedentes familiares $(77 \%)$. O estudo realizado por Coello em 2003, com 153 estudantes de medicina investigando a história familiar para DAC, AVC e dislipidemia, representou $94,8 \%$ dos alunos com antecedentes familiares superando os dados do nosso estudo. Observamos valores muito elevados na prevalência do sedentarismo (55\%). No entanto em um estudo transversal, em adultos jovens de uma universidade privada (SP) foram avaliados vários FR entre os quais o sedentarismo cuja prevalência manteve-se em $78,9 \%$. Estudos tem demonstrado que a quantidade do exercício físico, produz efeitos benéficios sobre a função cardiovascular, protegendo o miocárdio do vasoespasmo, reduzindo os FR para DAC (13).

Quando se avalia a CA da amostra, percebe-se que $75 \%$ apresentam risco aumentado para DCV e que ele esteve presente somente na população feminina. $\mathrm{O}$ estudo de Lima et al. (2003) mostra que a presença de lipemia pós-prandial aumentada se associou ao acúmulo da gordura na região abdominal e a níveis mais baixos de HDL-colesterol mesmo quando a trigliceridemia de jejum estava "normal" caracterizando que a obesidade central é um forte indicativo de doença cardiovascular (14).
O estresse esteve presente em 53,8\% da amostra, com uma prevalência direta entre os trabalhadores lotados em unidades de alta complexidade $(56 \%)$. Conforme a classificação de Lipp as cinco fases do estresse, podemos perceber que o profissional enfermeiro apresentou um percentual maior em quatro fases, sendo um dado preocupante por se tratar dos estágios finais de estresse. Estes achados vem ao encontro de estudos que associam os níveis de estresse com grau de responsabilidade e sobrecarga de trabalho que um enfermeiro exerce, produzindo sintomas físicos e psicológicos, bem como um aumento do níveis de colesterol e do tônus cardíaco (15).

Estudos demonstram que a relação entre prazer e sofrimento entre profissionais de enfermagem, ocorre quando há a possibilidade de demonstrar afeto e dedicação às atividades do cuidado. No entanto quando há evidência da morte em paciente crítico, torna-se evidente uma situação mais estressante de serem enfrentadas pelos profissionais $(16,17)$.

Em um outro estudo, Lautert identificou as fontes geradoras de estresse nos enfermeiros, e o que poderiam acarretar sobre sua saúde. Neste estudo, exploratório descritivo, com 33 enfermeiros, as alterações cardiovasculares foram as que tiveram maior incidência nestes auto relato, junto com problemas gastrintestinais (18).

Ao associarmos o estresse com o IMC, nossos resultados demonstraram que os sujeitos de pesquisas com IMC acima do normal, também apresentavam níveis de estresse elevado. Estudos suecos liderados pelo professor Björntorp vêm, há alguns anos, mostrando evidências de que as pessoas sob estresse continuado, que estão sempre sob pressão, terminam modificando o padrão de secreção do hormônio cortisol (hormônios de resposta ao estresse), aumentando a fome e a deposição de gordura na região central do corpo (19).

As condições de saúde de um indivíduo manifesta-se na maioria das vezes através de 
suas condições físicas e/ou psíquicos ocasionados pelas condições sócio-econômicas e culturais (20).

\section{CONSIDERAÇÕES FINAIS}

Mediante a análise dos resultados, o presente estudo permitiu concluir que os FR mais freqüentes encontrados nesta população foram sedentarismo, história familiar, aumento da circunferência da cintura abdominal, estresse, indicando a necessidade de programas educativo junto as instituições visando despertar maior conscientização nos trabalhadores em saúde. $\mathrm{O}$ fato de nenhum resultado apresentar significância estatística, no entanto, com alta prevalência em níveis limítrofes não invalida os dados, porém em se tratando de uma população jovem, o seguimento do estudo poderá trazer resultados surpreendentes. Neste contexto, os programas a serem adotados, devem ser permanentes, sistematizados iniciando já na infância, visando auxiliar a promoção à saúde e prevenção de doenças crônico-degenerativas em busca do desenvolvimento de estilos de vida mais saudáveis. É de suma importância a realização de outros estudos onde possa haver outras formas de repensar em qualidade de vida ao trabalhador de saúde.

\section{REFERÊNCIAS}

1. Smeltzer SC, Bare B. Brunner \& Suddart: Tratado de Enfermagem Médico - Cirúrgica. $9^{a}$ ed. Rio de Janeiro: Guanabara Koogan; 2003.

2. Guedes D, Guedes J, Barbosa D, Oliveira J, Stanganelli L. Fatores de risco cardiovasculares em adolescentes: Indicadores biológicos e comportamentais. Arq Bras Cardiol. 2006; 86(6): 439-450.

3. Elias M, Bolívar M, Fonseca F, Martinez
T, Angelini J, Ferreira C, et al. Comparação do Perfil Lipídico, Pressão Arterial e aspectos nutricionais em Adolescentes filhos de hipertensos e normotensos. Arq Bras Cardiol. 2004; 82(2): 139-142.

4. Ministério da Saúde. Fundação Nacional de Saúde. DATASUS. Informações de Saúde. 2002. Encontrado em http//www. datasus.gov.br. Acceso el 29 de abril de 2002.

5. Sociedade Brasileira de Cardiologia SBC. Sociedade Brasileira de Hipertensão - SBH. Sociedade Brasileira de Nefrologia - SBN. V Diretrizes Brasileiras de Hipertensão Arterial. 2006. Encontrado em http://publicacoes.cardiol.br/ consenso/2006/VDiretriz-HA.pdf. Acceso en octubre 2009.

6. Pitanga F, Lessa I. Indicadores antropométricos de Obesidade como instrumento de Triagem para risco coronariano elevado em adultos na cidade de Salvador-Bahia. Arq Bras Cardiol. 2005; 85(1): 26-31.

7. Sociedade Brasileira de Cardiologia SBC. III Diretrizes Brasileiras sobre Dislipidemia e Diretriz de Prevenção do Departamento de Aterosclerose da Sociedade Brasileira de Cardiologia. Arq Bras Cardiol. 2001; 77(3): 1-48.

8. Carlini E, Galduróz J, Noto A, Nappo S. I Levantamento domiciliar sobre uso de drogas no Brasil. São Paulo: Centro Brasileiro de Informações sobre Drogas Psicotrópicas; 2001.

9. Gross J, Silveiro S, Camargo J, Reichelt AJ, Azevedo M. Diabetes Melito: diagnóstico, classificação e avaliação do controle glicêmico. Arq Bra Endocrinol Metab. 2002; 46(1): 16-26.

10. Lipp M. Manual do Inventário de Sintomas de Estresse de Adultos de Lipp (ISSL). São Paulo: Casa do Psicólogo; 2000.

11. Pierin AME. Hipertensão Arterial - Uma proposta para cuidar. São Paulo: Manoele; 2004. 
12. Fonseca TMG. De mulher à enfermeira: conjugando trabalho e gênero. In: Lopes MJ, Waldow VR, Meyer D. Gênero \& Saúde. Porto Alegre: Artes Médicas. 1996: 63-75.

13. Coelho V, Caetano L, Liberatore R, Cordeiro J, Souza D. Perfil Lipídico e Fatores de Risco para Doencas Cardiovasculares em estudantes de Medicina. Arq Bras Cardiol. 2005; 85(1): 57-62.

14. Rabelo L, Viana R, Schimith M, Patin R, Valverde M, Denadai R, et al. Fatores de Risco para Doença Aterosclerótica em Estudantes de uma Universidade Privada em São Paulo-Brasil. Arq Bras Cardiol. 1999; 72(5): 569-574.

15. Lima J, Nóbrega L, Nóbrega M, Bandeira F, Souza A. Dislipidemia pós-prandial como achado precoce em indivíduos com baixo risco cardiovascular. Arq Bras Endocrinol Metabol. 2002; 46(3): 249-254.
16. Peiró JM, Salvador A. Control del Estrés Laboral. Madrid: Eudema; 1993.

17. Gutierrez B, Ciampone M. Profissionais de Enfermagem frente ao processo de morte em cuidados de terapia intensiva. Enfermagem. Acta Paul Enferm. 2006; 19(4): 456-461.

18. Lautert L, Chaves EHB, Moura GMSS. O estresse na atividade gerencial do enfermeiro. Dissertação de mestrado. Porto Alegre: UFRGS; 1996.

19. Wallerius S, Rosmond R, Ljung T, Holm G, Björntorp P. Rise in morning saliva cortisol is associated with abdominal obesity in men: a preliminary report. J Endocrinol Invest. 2003; 26(7): 616619.

20. Castro M, Rolim M, Mauricio T. Prevenção da hipertensão e sua relação com o estudo de vida de trabalhadores. Acta Paul Enferm. 2005; 18(2): 184-189. 